\title{
INVESTIGATION OF THE ACTIVE IONS DISTRIBUTION IN LASER CRYSTALS USING HIGH RESOLUTION SPECTROSCOPY
}

\author{
N. Peldetier-Allard and R. Pelletier \\ Laboratoire A. Cotton, C.N.R.S. II, bât. 505, 91405 Orsay, France
}

Iligh resolution spectroscopy techniques are used to investigate the distribution of rare-earth ions inside crystal hosts and their transfer properties. Examples concerning two typical laser materials are treated.

PACS numbers: 78.45.+h, 78.50.Ec, 78.55.Hx

\section{Introduction}

Research on solid state lasers presently knows an important development since they can produce compact and efficient emitting systems. They are made of ordered crystals (YAG, titanium sapphire) or of completely disordered materials (glasses). The fluorescence consists of narrow lines or of broad bands leading to a possible tunability of the laser emission. In any case, the spectroscopic properties of the laser material are related to the condition of the lasing performances. Since these arc related to the distribution of the active ions inside the host, we have taken interest in the determination of the location of doping rare-earth ions in various matrices.

The investigation is carried out through the study of the profiles of the optical absorption and fluorescence lines, which contain a large amount of information about the microstructure. Since the ions are not randomly distributed, and therefore are not submitted to identical crystal fields, several lines correspond to the same transition. A continuous distribution of resonance frequencies leads to the inhomogeneous lineshape.

This paper presents the examples of two typical cases:

- In the first case there is an additional discrete spectrum due to ions in perturbed sites, pairs or clusters; the lines are buried under the inhomogeneous main resonance line, or they appear as a satellite structure.

- The second example concerns the case where the distribution of local environments leads to a limited number of slightly shifted broad lines, and the spectral profile is the envelope over these lines.

The simultaneous study of the energy transfer processes leads to additional information concerning the efficiency of the various possible excitations. 


\section{Experiment}

The experiments are performed using the techniques of high resolution spectroscopy. The spectrometer is a grating spectrometer (Jobin Yvon THR) for which the resolution limit is $0.2 \mathrm{~cm}^{-1}$. It is used for conventional absorption and site selective fluorescence spectroscopy. Fluorescence is induced by a single frequency continuous wave dye laser (Spectra Pliysics ring cavity model 380) pumped by an argon ion laser (Spectra Physics Ion Laser 2000).

Excitation spectra are obtained through a step by step scanning of the laser wavelength. A step size of $0.08 \mathrm{~cm}^{-1}$ is used, and the shape of the profiles is obtained by adjusting, for each step, the intensity of the laser beam to a reference value. The intensity variations of the fluorescence are observed on definite emission lines.

A very accurate calibration $(0.02 \AA)$ of the laser wavelength is obtained by use of a lambdascope [1]. 'This system, which is convenient for both $\mathrm{cw}$ and pulsed lasers, makes use of the method of overlapping orders. A 50 lines $/ \mathrm{mm}$ grating spectroscope illuminated by a neon lamp gives about 1500 references lines in the visible range. Around the 55th order it provides two neon reference lines in the immediate vicinity of any laser radiation so that the wavelength is obtained by a simple linear interpolation.

The existence of a reabsorption of some lines has to be taken into account since it induces important changes in the excitation profiles. The phenomenon appears when the corresponding electronic transitions possess important oscillator strengths and when there is a large number of ions able to absorb the emitted radiation. Therefore only the main electronic resonance lines are concerned, and the satellite lines, which are relative to ions much fewer in number, are no longer hidden in the wings of the main line and can be recorded. The amount of reabsorption can be modified by focusing the laser beam in the crystal at varying depth from the front surface (fluorescence being observed at right angle to the beam direction).

In order to reduce the linewidths and consequently improve the resolution, all the spectra are recorded with the sample cooled at $4 \mathrm{~K}$ by a static exchange gas in a continuous flow cryostat (CF 204 Oxford Instruments).

\section{Method of analysis}

The lineshapes result from the addition of contributions from ions residing in different environments, therefore having their energy levels shifted. In order to extract the behaviour of specific subsets within the ensemble, the reconstitution of the profiles is computed using the Gaussian approximation, since it is generally assumed that inhomogeneous broadenings give rise to Gaussian lineshapes.

Inside a line relative to one electronic transition, each component corresponds to a specific perturbation, and its shift is obviously independent of the type of the recorded spectrum (absorption, excitation, fluorescence). Therefore the energy of any gaussian component is a fixed parameter in all the reconstitutions. On the contrary the intensities and linewidths are adjustable parameters which depend upon the type of the spectrum and the concentration of the doping ion. 
The method revealed itself to be a very sensitive one since even small changes in the value of any of the parameters introduce drastic effects on the calculated profiles. It has led to very accurate fits of the calculated to the experimental spectra.

\section{Nd in Er doped YAlO}

The diagram of the transitions involved in the study is in Fig. 1. A comparison between $\mathrm{Nd}$ spectra obtained with a $0.5 \% \mathrm{Nd}: \mathrm{YAlO}_{3}$ sample and a $0.5 \% \mathrm{Nd}$ : $\mathrm{Er}_{0.05} \mathrm{Y}_{0.95} \mathrm{AlO}_{3}$ sample shows spectacular differences (Fig. 2). In the $589 \mathrm{~nm}$ and $875 \mathrm{~nm}$ absorption lines of the codoped sample, the satellite spectra are no more observable. In the excitation spectrum of the $589 \mathrm{~nm}$ line, the reabsorption of the main line is drastically reduced. As a matter of fact, these features exhibited on the $\mathrm{Nd}$ spectra obtained with the $5 \times 10^{-3} \mathrm{Nd}$ : Er,YAlO sample are found on the spectra which have been obtained with a 100 times less concentrated Nd:YAlO sample.

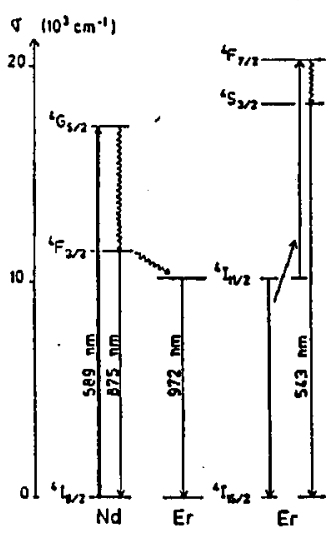

Fig. 1. Nd, Er relevant transitions in the YAlO study.

The origin of the codoping effects has been investigated through an analysis of the excitation profile of the $589 \mathrm{~nm}$ line. Monochromatic excitation spectra were monitored on various specific fluorescence lines. Figure 3 exhibits different excitation profiles induced by the scanning of the same absorption profile. Spectra (a) and (b) were recorded on the $\mathrm{Nd} 875 \mathrm{~nm}$ fluorescence line, the emitting ${ }^{4} F_{3 / 2}$ (1) level being populated through' a non radiative deexcitation. Spectra (c) and (d) were recorded on Er fluorescence lines, which imply the existence of a $\mathrm{Nd} \rightarrow \mathrm{Er}$ energy transfer. Moreover the emitting level of spectrum (d) is populated after an up-conversion process.

It has to be noticed that the spectra (b), (c) and (d) were obtained under identical geometrical conditions (position of the laser beam inside the sample for a weak reabsorption). Spectrum (a) was obtained with the laser beam as far as possible from the front surface for a maximum reabsorption, and is represented here in order to illustrate the errors that can be induced by the existence of 

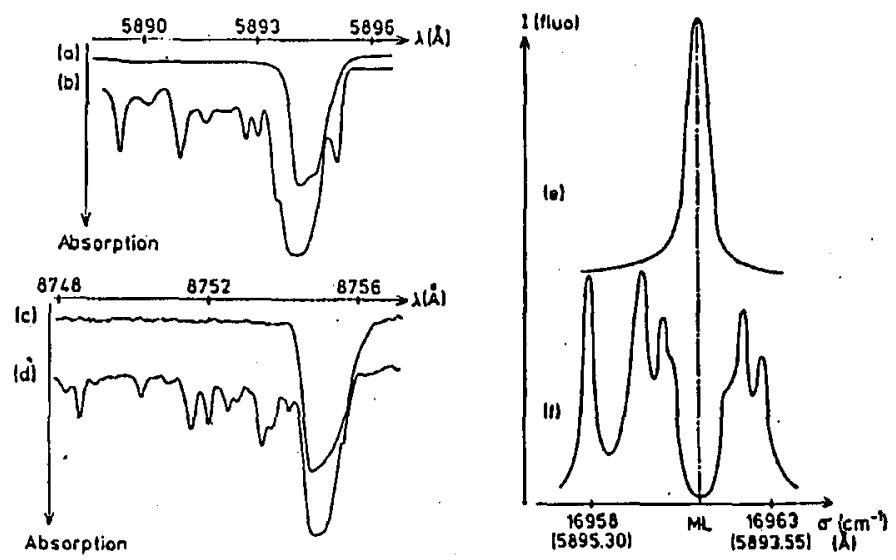

Fig. 2. Absorption (a, b, c, d) and excitation (e, f) spectra of $0.5 \% \mathrm{Nd}$ in $\mathrm{Er}_{0.05} \mathrm{Y}_{0.95} \mathrm{AlO}_{3}(\mathrm{a}, \mathrm{c}, \mathrm{e})$ and $\mathrm{YAlO}_{3}(\mathrm{~b}, \mathrm{~d}, \mathrm{f})$.

reabsorption; it appears to be a bump which can be mistaken for a satellite, and the maxima of the profiles are different $\left(16951.50 \mathrm{~cm}^{-1}\right.$ in $(\mathrm{a}), 16951.15 \mathrm{~cm}^{-1}$ in (d)).

The different profiles of Fig. 3 have been reconstituted (Fig. 4), assuming that the main line and the satellites liave the same linewidth. The circles represent the experimental profiles of Fig. 3, the solid lines represent the Gaussian profiles and the sum of their contributions. The intensities are in arbitrary units and normalised separately in each spectrum. The energy scale is the same for the four spectra of Figs. 3 and 4.

The variations in the relative intensities of the various components (only the three more important satellites will be considered) allow some conclusions, given that:

- The main line corresponds to Nd ions randomly distributed in regular sites;

- A satellite line corresponds to $\mathrm{Nd}$ ions in an intersital sites in a distorted site, generally due to the proximity of another impurity, different or identical;

- An up-conversion process is assumed to be due to an energy exchange between two ions, identical in most cases. Therefore its probability increases with the proximity of the ions.

The intensity of the satellite on the high energy side is important in (b), considerably reduced in (c), and zero in (d). The unefficient $\mathrm{Nd} \rightarrow$ Er energy transfer means an important separation between the corresponding $\mathrm{Nd}$ ions and any $\mathrm{Er}$ ion. Moreover, the few excited Er ions do not undergo an up-conversion process, which means that they are isolated from other $\mathrm{Er}$ ions. The satellites on the low energy side exhibit an opposite behaviour, which means that the corresponding Nd ions are close to $\mathrm{Er}$ ions. The up-conversion process on the $\mathrm{Er}$ ions being very efficient means that the energy is transferred on to two Er ions, which implies the existence of four-ions clusters. As for the Nd ions relative to the main line, they transfer 

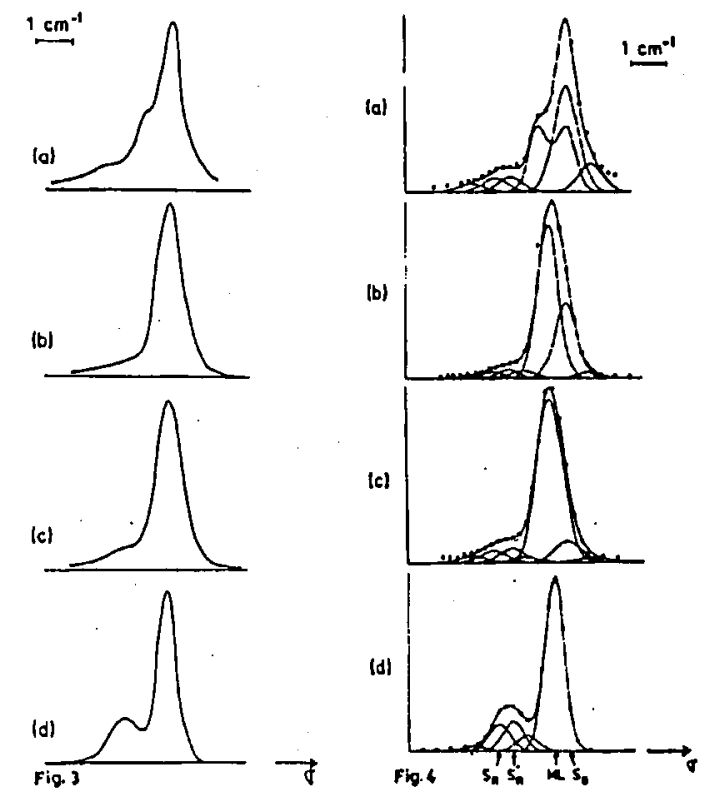

Fig. 3. $589 \mathrm{~mm}$ excitation spectrum monitored on the $875 \mathrm{~nm} \mathrm{Nd}$ line $(\mathrm{a}, \mathrm{b}), 972 \mathrm{~nm}$ Er line (c) and $543 \mathrm{~nm} \mathrm{Er} \mathrm{line} \mathrm{(d).}$

Fig. 4. Reconstituted profiles.

their energy to Er ions which exhibit the up-converted emission usual for a crystal $5 \%$ concentrated.

In short, in a YAlO crystal, the presence of erbium has the following effects on the neodymium spectra:

- The satellite spectrum removed from the main line is no longer visible.

- The Nd emission is weak and the energy transfer on Er is particularly efficient.

Therefore it can be assumed that there are very few $\mathrm{Nd}$ ions distributed in perturbed sites, near another $\mathrm{Nd}$ or any impurity of the host and that most of the $\mathrm{Nd}$ ions are in the vicinity of Er ions.

To conclude, the analysis of the excitation profiles of a $\mathrm{Nd}$ transition in an Er codoped YAlO crystal has shown that the quenching of the $\mathrm{Nd}$ fluorescence, already observed by Stalder et al. [2], has its origin in a non-random distribution of the Nd ions which substitute preferentially in the vicinity of the Er ions [3].

\section{5. $\mathrm{Nd}$ in gehlenite}

In $\mathrm{Ca}_{2} \mathrm{Al}_{2} \mathrm{Si}_{7}$, the cations are localized on three types of sites: a Thomson cube (TC) occupied by $\mathrm{Ca}^{2+}$, a regular tetrahedral site $\left(T_{1}\right)$ occupied by $\mathrm{Al}^{3+}$, a very distorted tetrahedral site $\left(T_{2}\right)$, where $\mathrm{Si}^{4+}$ and $\mathrm{Al}^{3+}$. ions are statistically distributed. The $\mathrm{Nd}^{3+}$ ions substitute $\mathrm{Ca}^{2+}$ in the $\mathrm{TC}$ sites, and the charge balance 
leads to the doped gehlenite formula $\frac{\mathrm{Ca}_{2-\mathrm{x}} \mathrm{Nd}_{\mathrm{x}}}{\downarrow} \underset{\mathrm{TC}}{\frac{\mathrm{Al}}{T_{1}}} \frac{\mathrm{Al}_{1+\mathrm{x}} \mathrm{Si}_{1-\mathrm{x}} \mathrm{O}_{7}}{\downarrow} T_{T_{2}}$ [4].

All the recorded Nd absorption and fluorescence spectral profiles exhibit broad lines with non Gaussian shapes, which is the evidence that several lines are buried under each profile. Previous to any reconstitution, the number of possible $\mathrm{Nd}$ sites has been determined.

It is assumed that only the nearest neighbours of the Nd ions have an effect upon the Nd crystal field. No eflect being expected from the $T_{1}$ tetrahedra occupied by $\mathrm{Al}$ ions exclusively, it is considered that the optical properties depend mainly upon the $\mathrm{Al}$ and $\mathrm{Si}$ random distribution in the $T_{2}$ tetrahedra.
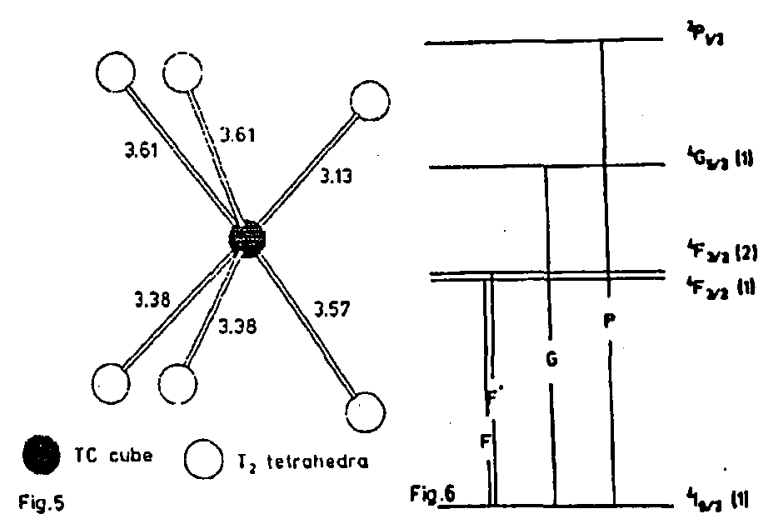

Fig. 5. Nd surrounding in gehlenite.

Fig. $6 \mathrm{Nd}$ relevant transitions in the gehlenite study.

The location of the nearest six $T_{2}$ tetrahedra surrounding one $\mathrm{Nd}$ ion is represented in Fig. 5. The number of possible $\mathrm{Al}-\mathrm{Si}$ distributions in these sites has been found to be twelve, by considering that the most probable repartition in the immediate vicinity of each $\mathrm{TC}$ is $50 \% \mathrm{Al}$ and $50 \% \mathrm{Si}$. Therefore it can be considered that the $\mathrm{Nd}$ ions are shared amongst twelve types of sites, and consequently that the various spectral profiles are the envelope of twelve Gaussian lines. Their intensities depend upon the Nd distribution probability, their shifts depend upon the perturbation, i.e. upon the $\mathrm{Al}$ and Si respective locations.

As a matter of fact, eight lines are sufficient to fit all the profiles, which means a reduced probability for four sites or similar crystal field effects for four pairs of sites. The diagram of the transitions involved in the study is in Fig. 6. The spectra shown in the following figures are from a $x=0.005$ concentrated sample. The circles represent the experimental profiles, the dotted and solid lines represent respectively the Gaussian profiles and the sum of their contributions. It has been assumed that all the Gaussian components inside a line relative to a particular transition have the same linewidth.

Figure 7 represents the $\sigma$ lines relative to the $P(a)$ and $F^{\prime}(\mathrm{b})$ transitions, 
the $\pi(c)$ and $\sigma(\mathrm{d})$ lines relative to the $F$ transition. Figure $7(\mathrm{e}, \mathrm{f})$ represents only the low energy side of the $\pi$ and $\sigma$ lines relative to the $G$ transition: the high energy side is overlapped by the line relative to the second Stark level of the ${ }^{4} G_{5 / 2}$ multiplet, and a total deconvolution is not possible due to the lack of symmetry of the spectral profiles in the studied crystal.
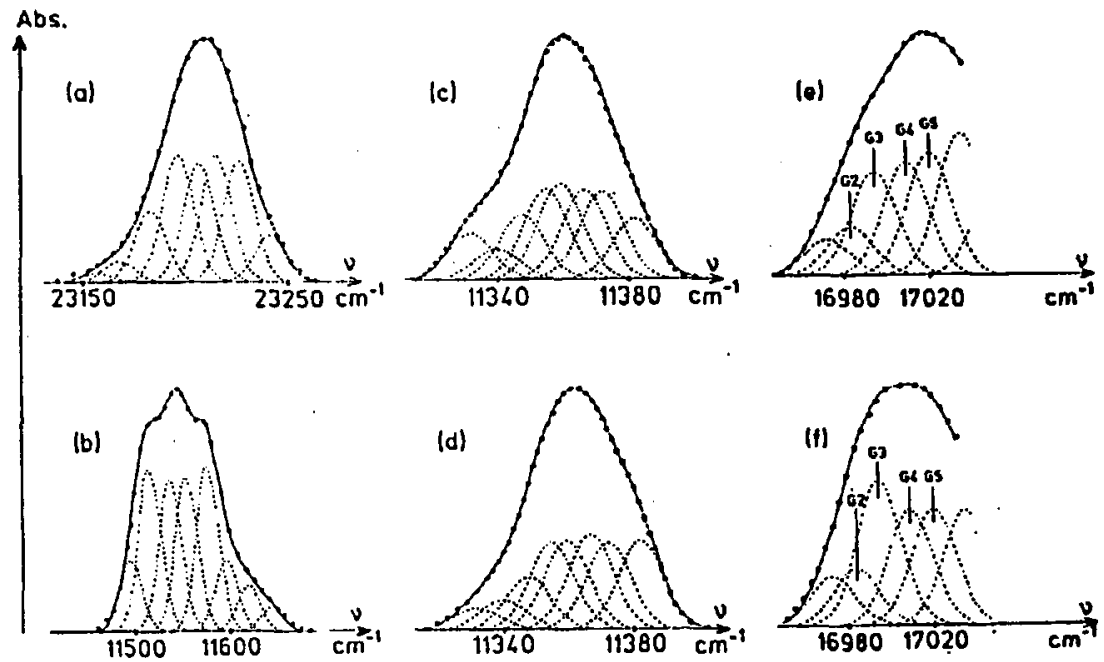

Fig. 7. $P$ absorption (a); $F^{\prime}$ absorption (b); $F \pi, \sigma$ absorption (c, d); $G \pi, \sigma$ absorption $(\mathrm{e}, \mathrm{f})$.

Fluorescence was induced by excitation on the maxima of the second to the fifth $G$ Gaussian components and observed on the $F$ line. For each energy four spectra were recorded, the exciting beam and the emitted light being both polarized, parallel $(\pi)$ or perpendicular $(\sigma)$ to the crystal axis. The example in Fig. 8 concerns the $G 4$ excitation. It has to be noticed that the four presented spectra and the two spectra of Fig. $7-\mathrm{e}, \mathrm{d}$ actually are fitted with eight components all having the same energy. So are the twelve other fluorescence spectra, whatever their shape is, and also the eighteen spectra recorded in identical conditions with a $x=0.02 \mathrm{Nd}$ concentrated sample.

The interpretation of the whole of the experimental data has led to interesting results:

- The absorbed energy does not remain localized on the excited ions, which implies the existence of energy transfers with concentration of the energy on preferential sites;

- The fluorescence line narrowing is weak, which implies the existence of a spectral diffusion inside each site;

- Some lines are completely polarized, which means that all Nd sites have identical sets of symmetry axes, opposite to glasses. 
- An increase in concentration does not involve any spectral change, except for a broadening of the lines.
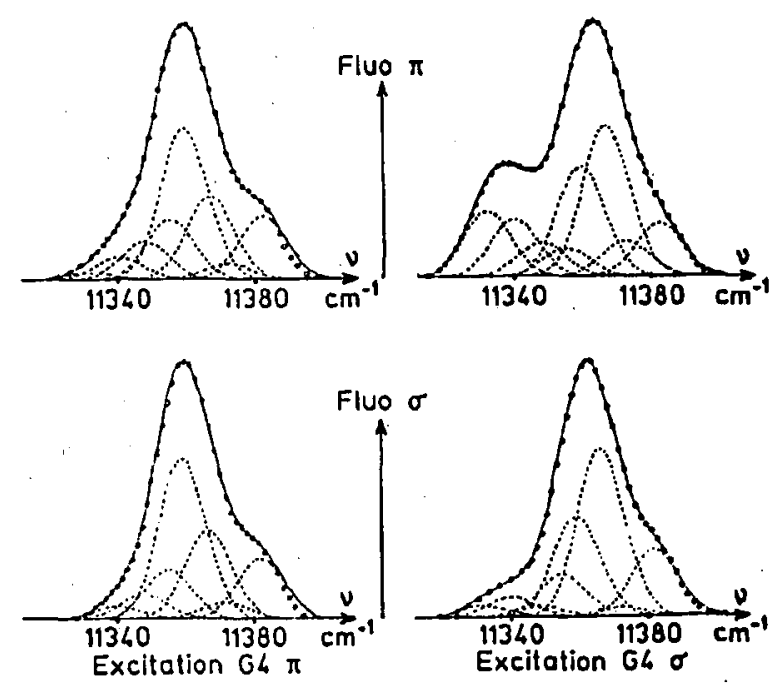

Fig. 8. G4 excited $F$ fluorescence.

To conclude, gehlenite is a laser material intermediate between ordered crystals and glasses. The analysis of the absorption and fluorescence profiles of $\mathrm{Nd}$ has shown that the active ions are distributed in definite sites equally oriented in space, which confers a crystal character to the matrix. Meanwhile the corresponding lines are broad and overlap, which leads to large inhomogeneous linewidths, and therefore to a possible tunability of the laser emission [5].

\section{Conclusion}

This paper presents the emitting properties of laser materials, in relation with the distribution of the active ions inside the host. The investigation is built on the profiles of the spectral lines recorded in different conditions: absorption, emission observed after a cascade process, an interionic transfer process or an up-conversion process, the interpretation being drawn from their reconstitution. Two examples illustrate the efficiency of the method.

\section{References}

[1] N. Pelletier-Allard, R. Pelletier, Rev. Sci. Instrum. 55, 1442 (1982).

[2] M. Stalder, M. Bass, J. Opt. Soc. Am. B 8, 177 (1991).

[3] N. Pclletier-Allard, R. Pelletier, C. Tiseanu, J. Lumin. 62, 257 (1994).

[4] B. Viana, A.M. Lejus, D. Saber, N. Duxin, D. Vivien, Opt. Mater, 3, 307 (1994).

[5] A.M. Lejus, N. Pelletier-Allard, R. Pelletier, D. Vivien, submitted to publication. 